\title{
The impact of the COVID-19 pandemic on children's mental health and wellbeing, and beyond: A scoping review
}

\author{
Jane Spiteri ${ }^{1}$
}

\begin{abstract}
The major threat of COVID-19 has become a priority to education and health systems worldwide. This scoping review reports on, and analyses, the literature pertaining to the impact of the COVID-19 pandemic on children's mental health and wellbeing, and the resources needed to assist them in these difficult times. The findings of this literature review point out the impacts of the pandemic on the mental health and wellbeing of children hailing from different socio-economic backgrounds, as well as the impacts on families and schools. They also highlight how lockdown, quarantine, social distancing, social media and the measures needed to prevent the spread of infection can negatively affect children's mental health and wellbeing. Consequently, cautionary measures that minimise these impacts on children, and recommendations for policy, research and practice are discussed.
\end{abstract}

\author{
Article History \\ Received: 26 March 2021 \\ Accepted: 22 June 2021 \\ Keywords \\ Children; COVID-19; \\ Pandemic; Mental health; \\ Wellbeing
}

\section{Introduction}

Historically, major disease outbreaks, such as pandemics, have burdened humanity. Pandemics are infectious disease outbreaks that threaten human health on a global scale (Fong \& Iarocci, 2020). On March 11th, 2020, the World Health Organization (WHO) declared the novel coronavirus (SARS-CoV-2), known as COVID-19, a pandemic (WHO, 2020). In response to this declaration, many countries have imposed lockdowns and other social distance measures to prevent the spread of the coronavirus within the community (Fong \& Iarocci, 2020).

Quarantine and disease contamination measures are considered unpleasant by most (Brooks et al., 2020), and can be traumatising to many (Sprang \& Silman, 2013). This is mostly because lockdown and social isolation are especially hard on individuals, particularly for children and their parents, and these have been shown to trigger symptoms of mental health issues, such as depression and anxiety (Organisation for Economic Co-operation and Development [OECD], 2020a). This has led to the recent growing interest in the psychological manifestations of the pandemic (Ashikkali, Carroll, \& Johnson, 2020; Sprang \& Silman, 2013). Indeed, strong evidence confirms the profound psychological impacts lockdowns and isolation on humanity (Brooks et al., 2020; Garcia de Avila et al., 2020; Hawke et al., 2020; Roy et al., 2020; Yue, Zang, Le, \& An, 2020). Prior research has consistently shown that families who were either isolated, or quarantined, during the H1N1 pandemic, in the United States, experienced post-traumatic stress disorder (PTSD) symptoms and anxiety (Sprang \& Silman, 2013). Similarly, following a review of the psychological impact of previous quarantines during SARS, the 2009 and $2010 \mathrm{H} 1 \mathrm{~N} 1$ influenza pandemic, MERS, Ebola, and equine influenza, Brooks et al. (2020) reported the harmful psychological effects of quarantine, which included symptoms of PTSD, confusion, and anger. Simply put, quarantine, fear of infection, frustration, boredom, financial loss, inadequate information, stigma and lack of support, can have long-lasting effects on individuals (Brooks et al., 2020).

Few months into the COVID-19 pandemic, humanity has come to the realisation that disease

${ }^{1}$ University of Malta, Faculty of Education, Department of Early Childhood and Primary Education, Msida, Malta, e-mail: jane.spiteri@um.edu.mt, ORCID: https://orcid.org/00000001-6625-2372 
prevention measures, such as lockdown and social distance, coupled with the direct effects of the pandemic, such as illness and mortality, and the indirect effects on the economy, workplace, schools/day care and social life, have generated significant stress on communities, leading to a multitude of psychological challenges to many, including children (United Nations Educational, Scientific and Cultural Organization [UNESCO], 2020a, 2020b, 2020c). Major mental health issues reported by children during a pandemic included stress, anxiety, depressive symptoms, insomnia, denial, anger and fear (Roy et al., 2020). This is not surprising considering the bidirectional relationship between mental health issues, such as depressive disorders and anxiety, and poverty, destressing life events and physical illnesses (WHO, 2017).

Understanding the complexity of the psychological and mental health impacts of COVID-19 on different segments of the population is vitally important if children (from birth to 17 years of age) are to be provided with the appropriate support to safeguard their wellbeing. As a vulnerable group of society, children are experiencing the psychological impacts of the COVID-19 pandemic. These can potentially have longstanding implications for their health outcomes (Kang, Lim, Ragen, Tan, \& Aishworiya, 2020), and their social and economic future (Prime, Wade, \& Browne, 2020). We now know that the number of new infections and the death rate are likely to increase unless the community has been vaccinated. Therefore, understanding and addressing the stressors caused by the pandemic are essential first steps towards promoting and protecting the wellbeing of children (Prime et al., 2020).

\section{The Present Study}

Despite the dire impact of COVID-19 on humanity, particularly on children, there is paucity in research related to the effect of the pandemic on children's mental health and the resources needed to assist children in these difficult times (Fong \& Iarocci, 2020). Furthermore, "the precise extent to which COVID19 is shaping children and family functioning is largely unknown" (Prime et al., 2020, p. 639). It is important for professionals working with children and families to understand the impact of COVID-19 on children and how these are influenced by family dynamics (Prime et al., 2020).

This paper fills this gap in the literature by drawing on the literature to describe the mental health issues experienced by children (from birth to 17 years of age) during the COVID-19 pandemic. The preliminary observations reported here serve as an informed basis to understand some of the impacts brought about by the uncertainly and the disruption caused by COVID-19. The primary focus is on children's wellbeing and the characteristics that may heighten the risk of the negative consequences of the pandemic. As such, this paper draws on the literature across topic areas, including education, health and crises, to examine the potential consequences (short- and long-term) of the COVID-19 pandemic on children's mental health within a context. In doing so, it presents a unique perspective, written from a particular part of the Western world, and a particular position, in relation to children's wellbeing during the COVID-19 pandemic. At the same time, it acknowledges that it is revealing experiences of the pandemic that might be strikingly different to some as it leaves out the perspectives and shared experiences (linguistic, cultural and geographical), voices and particular challenges which are uniquely shared by other communities from different parts of the world. Most striking though, is the recognition of the global impact of the pandemic on children.

In doing so, this paper provides a baseline of the literature and research moving forward, by synthesising currently available literature from a limited number of publications. More importantly, this paper initiates a dialogue about how unforeseen local and global crises, such as pandemics, might be managed in the future. In saying that, the author is mindful that drawing recommendations for children can be problematic as any recommendations made are likely to be influenced by context. Importantly though, when considering any recommendations and applying them to differing contexts, these need to be challenged and adapted to the environment in which it is being implemented. Finally, research strengths, gaps and implications are discussed. 


\section{Method}

In light the above, the current study adopted a scoping review of the literature to inform research related to the impact of COVID-19 on children's mental health. Scoping review of the literature provides the framework for the identification and analysis of this article (Arksey \& O'Malley, 2005; Levac, Colquhoun, \& O'Brien, 2010). A scoping review assesses the potential size and scope of the availability, and extent, of the literature related to particular research questions (Grant \& Booth, 2009). Like systematic reviews, scoping reviews are useful for addressing research questions, in methodical and replicable ways (Arskey \& O’Malley, 2005).

A goal of the current study is to illustrate the mental health issues children experience during the pandemic. Hence, a scoping review methodology was considered optimal to address the broad questions guiding this paper, while making room for the inclusion of various publications. Furthermore, a scoping review allows for reflection on, and reconsideration of, articles relevant to include. This is highly desirable and relevant when considering the articles included in this review.

\section{Identifying the Research Questions}

The present study reports findings of a scoping review of the literature published during the COVID19 pandemic, between 2020 and 2021. İt was conducted to examine contemporary knowledge about the impact of the COVID-19 pandemic on children's mental health and wellbeing. Specifically, it aimed to map the different ways in which children's mental health and wellbeing have been impacted during the COVID19 pandemic, to help advance knowledge and educational practices. Consequently, this review addressed the following questions: (1) What is known about the impact of COVID-19 on children's mental health? (2) What prevention measures or procedures can be implemented in schools and at home?

\section{Identifying Relevant Studies}

Consistent with Arksey and O'Malley's (2005) recommendations, in this scoping review, broad search criteria were applied to identify studies related to the impact of the COVID-19 pandemic on children's mental health and wellbeing. Key databases were identified and searches were performed in each of the following databases: HyDi, ERIC, ProQuest Social Sciences, PsycInfo, PubMed, SCOPUS, Social Work Abstracts, and Web of Science. The search strategy included a combination of key words and subject headings that were conjoined using the Boolean operation AND/OR. Keywords included mental health, wellbeing, children, school, family, COVID-19, and pandemics. In this scoping review, "children" refers to all minors, from birth up to 17 years of age.

An iterative process influenced the article search process, where the diverse impact of the COVID19 pandemic on children's mental health and wellbeing have been considered (Arksey \& O'Malley, 2005). Inclusion criteria were that each study was: (a) published between 2020 and 2021; (b) related to the impact of the COVID-19 pandemic on children's mental health and wellbeing; (c) electronic sources; and, (d) published in English. Exclusion criteria were: (a) articles detailing intervention studies not related to the COVID-19 pandemic; (b) editorials; (c) opinion articles; (d) articles focussing on youths and adults; and (e) abstract not provided.

\section{Study Selection}

Since literature from diverse disciplines was considered for this scoping review, there was a number of articles that made room for comparisons and contrasts, a decision that offered balance between the number of articles found and the issues of feasibility of this scoping review (Levac et al., 2010). In line with a scoping review methodology, all relevant literature was included during the research design process. A decision was made to limit articles to those using data collected in 2020 or later, to ensure that only studies related to COVID-19 were included.

\section{Charting the Data}

An Excel worksheet was used to categorise the data. The collected data were charted in the Excel table, in the following categories: (a) an annotated bibliography of each article; (b) year of publication; (c) 
type of study; (d) database; (e) discipling of the first author; (f) participants and their age; (g) the impact of the COVID-19 pandemic on the participants' mental health and wellbeing; (h) authors' assumptions about their findings; (i) abstract of the study; and, (j) country of origin.

\section{Collating, Summarising and Reporting the Results}

Using the Excel table described above, the charted data were compared and contrasted by two reviewers who were not involved in the data collection. They examined the full-text review articles and excluded those that contained data collected before 2020. Their review enabled the rigour of the analysis. During this process, articles with similar perspectives were grouped together and descriptive summaries were written for each. Next, additional analysis led to the differences and similarities to emerge, both within each perspective and across categories. The final synthesis produced the impacts identified. A total of 22 articles were selected.

\section{Results}

The initial search strategy, shown in the flow diagram in Figure 1 below, outlined the Preferred Reporting Items for Systematic Reviews and Meta-Analysis (PRISMA), yielded a total of 150 articles. After removing duplicate and ambiguous articles, and going through the inclusion and exclusion criteria by relying on the titles and abstracts, 22 articles remained.

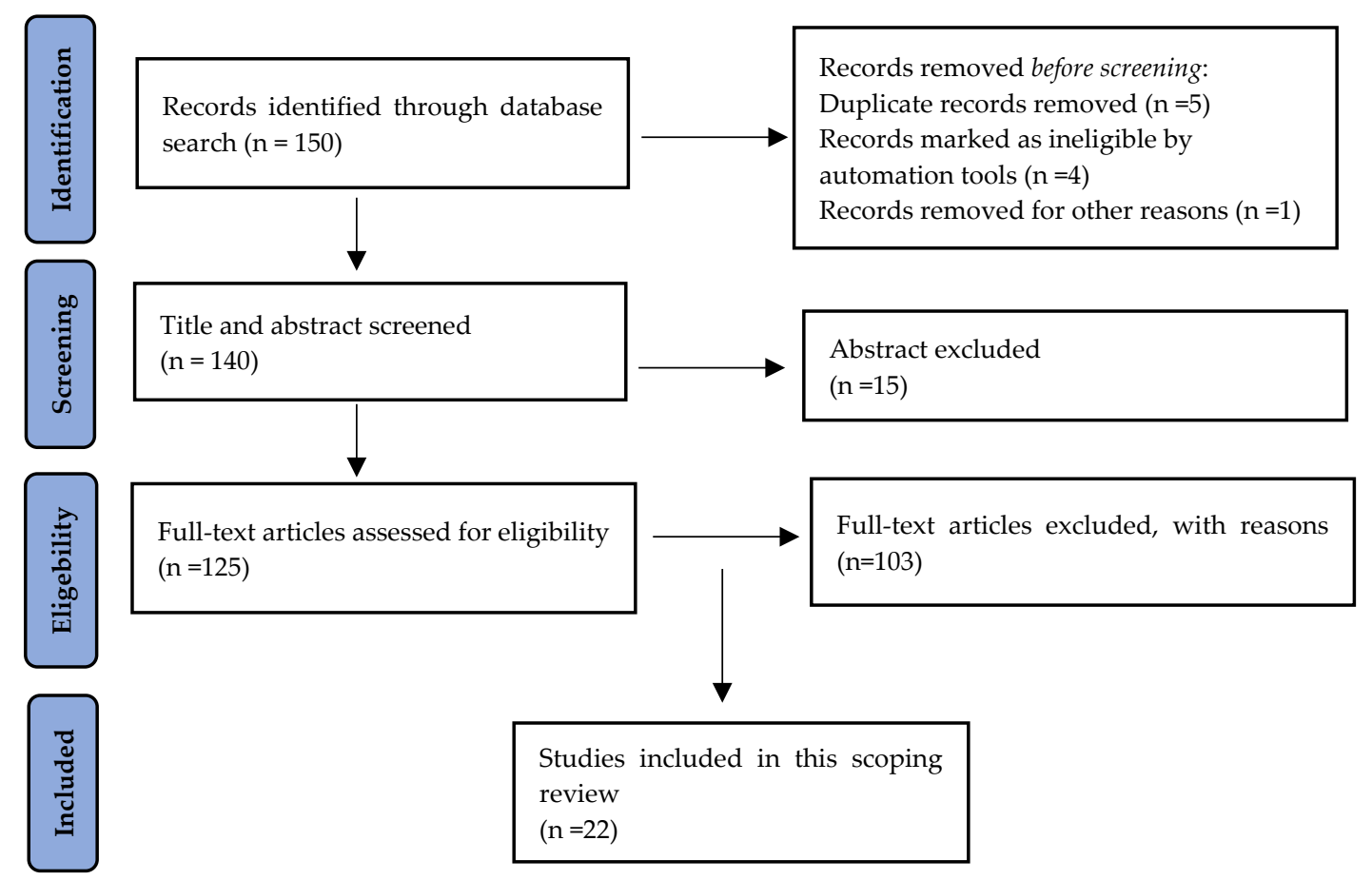

Figure 1. Preferred Reporting Items for Systematic Reviews and Meta-Analysis (PRISMA) flow chart depicting the identification, screening, eligibility and inclusion of studies within this scoping review.

One limitation of this scoping study that is worth noting is the fact that since it relied only on electronic sources, relevant documents published in a different format, or the ones published at a later date, have been excluded. Another limitation is the fact that it only considered articles published in English, thus leaving out valuable research published in languages other than English.

\section{Discussion}

Given the link between mental health and physical health, both are affected during a pandemic, and both can impact children's wellbeing (Ashikkali et al., 2020; Fong \& Iarocci, 2020). Clearly, during the COVID-19 pandemic children have been exposed, directly and vicariously, to some form of psychological 
distress, leading many to become stressed and anxious. Hence, children's mental health and wellbeing merit some investigation.

This scoping review aimed to identify and critically review published literature related to the impact of the COVID-19 pandemic on children's (from birth to 17 years of age) mental health and wellbeing. The analysis of the 22 studies selected revealed eight broad categories: children's wellbeing; children with preexisting mental health conditions; family relationships; school relationships; socio-economic backgrounds; lockdown, quarantine and social distancing; social media; and, psychological support services. The approach to discussing the results of each category draws on the narrative review, a common strategy to reporting findings of scoping reviews (Grant \& Booth, 2009).

\section{Children's Wellbeing}

While research shows that children's physical health is less likely to be impacted by the COVID-19 virus (Bond, Dibner, \& Schweingruber, 2020; Castagnoli et al., 2020; Ludvigsson, 2020; Spaull, 2020), new evidence is starting to emerge indicating that in a situation characterised by constant change, such as a pandemic, children are reporting unpleasant symptoms. Recent research suggests that anxiety, depression and mood disorders have been the most common mental health issues reported by children during the COVID-19 pandemic (Courtney, Watson, Battaglia, Mulsant, \& Szatmar, 2020; Kang et al., 2020). In fact, an escalation in physical symptoms experienced by children, including tummy aches, headaches, feeling sick and panic attaches, have reported by medical professionals despite the fact that these were otherwise healthy children and had not been infected by the coronavirus. Anxiety in children is manifesting itself as physical symptoms because children are more vulnerable to the negative impact of sustained stressful situation (Courtney et al., 2020; Garcia de Avila et al., 2020). It likely that while social distance measures have been effective at reducing the number of new infections, this has led a rise in anxiety, depression and other ailment, even in children (Gromada, Rees, \& Chzhen, 2020; Parsons, 2020; Xie et al., 2020).

Most studies exploring the role of gender in the management of anxiety during the COVID-19 pandemic found that gender was a risk factor for depression and anxiety symptoms (Fong \& Iarocci, 2020; Yue et al., 2020). Preliminary findings indicate that girls and women were hit harder during the pandemic (Fong \& Iarocci, 2020; Yue et al., 2020). Perhaps this is because during the pandemic women had to cope with the stress created by the pandemic itself, and the care responsibilities bestowed on them (Yue et al., 2020). However, literature has emerged that offers contradictory findings. For example, while Garcia de Avila et al. (2020) reported that girls experienced higher levels of anxiety than boys, Yue et al. (2020) reported that age and gender were not predictors of psychological distress in children. Definite conclusions cannot be drawn yet especially since studies related to the impact of gender on the management of anxiety and wellbeing during the COVID-19 pandemic are scarce. In fact, this is an area that merits further investigation (Fong \& Iarocci, 2020).

\section{Children with Pre-Existing Mental Health Conditions}

To date, the literature on children with pre-existing mental health conditions has highlighted an increase in the prevalence of anxiety (Garcia de Avila et al., 2020; OECD, 2020a; Parsons, 2020; Roy et al., 2020). Research by Roy et al. (2020) has established that children with pre-existing mental health issues had harder time coping with school closure, and the lack of contact and recreational activities outdoors. Reasons for this included fear of the disease, stigma, lack of awareness of risk, diminished personal protection, confinement in psychiatric wards during the pandemic, discrimination, and fear of social isolation (Roy et al., 2020). Similarly, Hawke et al. (2020) confirmed the significant risk posed by the pandemic for the exacerbation of symptoms of distress, such as anxiety, which are both painful and debilitating, in children with pre-existing mental health issues.

Surprisingly though, the disruption caused by the pandemic has improved some of the pre-existing anxiety disorders in children, even if only temporarily. Courtney et al. (2020) found that children who had been affected by social and performance anxiety and agoraphobia before the pandemic, did better during the pandemic, even if such improvement may be short-lived. Courtney et al. (2020) explained that children 
with pre-existing anxiety disorders may have felt less anxious during lockdown because the events that caused them anxiety before, such as separating from their parents, were gone and they felt they were in a safe space. The fact that these children had to maintain social distance measures and had to stay away from school may have helped ease their school anxiety. Similarly, Papetti et al. (2020) found that children and adolescents with primary and chronic headache disorders reported a significant improvement in the prevalence, intensity and frequency of headache attacks during the lockdown compared to the previous two months. As school anxiety and effort diminished, headache improvement was reported, indicating that lifestyle modification, as in the case of school closure, represents the main factor impacting the course of mental health issues (Papetti et al., 2020). Overall, in these studies, a reduction in school-related stress as a result of school closure was the main factor explaining the general improvement in the participants' mental health issues. Therefore, in these cases, school closure helped to improve the children's mental health.

\section{Family Relationships}

Central to children's wellbeing is the influence of the family system and the interactions therein (Bronfenbrenner \& Morris, 2006). Parents are in a good position to help their children buffer everyday stress and help them manage their feelings effectively (Courtney et al., 2020; Yue et al., 2020). In fact, time and time again, research has indicated a correlation between parental mental health and children's wellbeing, suggesting that caregivers' mental health is important to protect children's wellbeing (Fong \& Iarocci, 2020). During a pandemic, parents play a crucial role in providing emotional and psychological support to help their children deal with the uncertainty of the crisis, particularly by providing a stable and secure environment (Fong \& Iarocci, 2020).

Since the COVID-19 pandemic has amplified the stress families are experiencing, high parental anxiety can be projected onto children, negatively impacting their wellbeing (Kang et al., 2020; OECD, 2020a; Parsons, 2020; Sprang \& Silman, 2013). The mounting rates of unemployment during the pandemic have caused an abrupt and major upheaval in the daily lives of many families. Parsons (2020) argued that during the first lockdown, parental stress and worries over financial insecurities, and the pandemic itself, even if unintentionally, have impacted children's understanding of disaster, family tragedy and their resiliency. Loss of employment is a significant life stressor that can take a psychological toll on individuals, and can take years to recover (Infurna \& Luthar, 2016). Economic adversity and job loss cause stress, a major risk factor for increased family violence, psychological and physical maltreatment, and child abuse (Fong \& Iarocci, 2020; Lawson, Piel, \& Simon, 2020; WHO, 2020; Yeasmin et al., 2020). Since these uncertainties, coupled with the extra burden on parents by the pandemic, limit the parents' abilities to meet their family's needs, parental mental health may suffer. As a result, parents may be less able to help their children deal with emotional issues (Courtney et al., 2020; Fong \& Iarocci, 2020; Pittinsky, 2020). Under such circumstances, child psychological problems are likely to worsen (Sprang \& Silman, 2013), a situation encapsulated in the shadow of fear caused by the fact that children were restrained from attending school and extra-curricular activities, meeting relatives and peers, for fear of contracting the coronavirus (Courtney et al., 2020; Kang et al., 2020; Parsons, 2020).

Strong evidence suggests that parental educational level could be conducive to lower levels of anxiety in children during the COVID-19 pandemic (Garcia de Avila et al., 2020; Yeasmin et al., 2020; Yue et al., 2020). In a study by Yue et al. (2020), parents with higher income and higher educational attainment were reported as having lower levels of psychological distress probably because these attributes provided the family with more resources to understand and deal with the stressors created by the pandemic. Similar findings have been confirmed by Garcia de Avila et al. (2020), who reported that highly educated parents seemed to be in a better position to offer a range of support to their children, such as talking and listening to children's concerns over COVID-19, and providing further information, safer environments and emotional support, thus helping in minimising the children's anxiety levels.

In contrast, Yeasmin et al. (2020) reported a higher percentage of mental health issues in children whose parents were highly educated. The authors attributed this incidence to the fact that highly educated 
parents were still expected to work despite the lockdown and they did not have time to meet their children's demands and nurture them. As a result, children of higher-income parents experienced more psychological distress than children of low-income parents (Yeasmin et al., 2020). Based on the findings Yeasmin et al. (2020) concluded that it is likely that parents who are able to manage their emotions and depression, will be good role models of resilience for their children. Nevertheless, what seems to be more important for children during a pandemic is that parents are able to model healthy ways of dealing with anxiety triggers, particularly during stressful times like a pandemic (OECD, 2020a). In addition, financial support and flexible childcare provisions could ease the burden on families, thus enabling them to cope with their responsibilities during the pandemic, by increasing the chances of better mental health outcomes (Fong \& Iarocci, 2020).

\section{School Relationships}

In an attempt to mitigate the spread of the coronavirus, many educational institutions worldwide were closed and learning has been transferred to home-based learning, which has been mostly carried out online. It is estimated that mass school closure has affected approximately $91.3 \%$, or 1.5 million students, worldwide (UNESCO, 2020c). This has created global repercussions, impacting economic, educational and health systems, and the lives of children worldwide (UNESCO, 2020a, 2020b, 2020c). Indeed, many education experts have sounded the alarm numerous times about the detrimental effects of school closure on children's learning (Martin et al., 2020; OECD, 2020b; UNESCO, 2020d; United Nations, 2020). It is now clear that school closure has caused major interruptions to children's learning, and perhaps even worse, it has impacted children's social and emotional wellbeing.

Anxiety in children has increased exponentially following school closure, particularly because it has influenced family structures and the children's daily routines (Kang et al., 2020). With school closure and lack of socialisation, children's sense of belonging to the school community may have been lost, unless they kept abreast with online learning and activities (OECD, 2020a). The lack of social contact is impactful for all students, particularly the most vulnerable, who may suffer from social exclusion, physical violence, lack of family support and proper housing, and food insecurity (OECD, 2020a).

Age has been found to be an influential psychological stressor experienced by children. Existing research confirmed that primary school children were stressed and anxious because of school closure, particularly because the resources which they usually had access to through schools were no longer available when schools closed (Yue et al., 2020). Aware of this need, the OECD (2020a) has called for education systems worldwide to ensure children's social and emotional wellbeing, especially the vulnerable. To address the disruptions in children's education, UNESCO (2020b) developed ten key recommendations for learning, including wellbeing and educational needs of children, the emotional needs of educators, and the need for common directions/guidelines for educational institutions, to ensure that learning remains relatively uninterrupted during the pandemic.

Eventually, schools reopened but nervousness about COVID-19 began affecting some children once more (Pittinsky, 2020). Educators have an important role to play in reducing children's anxiety during the pandemic by providing a sense of stability to help children process, adjust, and develop new coping skills to deal with the uncertainty amid rapid change. Towards this end, educators can discuss COVID-19 has changed their school routine, rather than dismiss children's emotions as this may increase anxiety. In this regard, Pittinsky (2020) suggested that educators can discuss with children what is known and what remains unclear about the pandemic, help them understand the risks of new infections, ask them how they are doing and feeling, and emphasis positive ways of taking care of themselves, their family and friends hand washing, mask wearing and physical distancing.

\section{Socio-Economic Backgrounds}

Socio-economic background has been identified as a major contributing factor to the way children have been impacted by the pandemic. The impact of the economic crisis brought about by the pandemic had a significant impact on the social and emotional wellbeing of many children worldwide (Courtney et 
al., 2020; Garcia de Avila et al., 2020; Kang et al., 2020; Keshri, 2020; Parsons, 2020). The impact of the pandemic on children living in inadequate housing in low-income neighbourhoods and those experiencing domestic violence has been profound (Garcia de Avila et al., 2020; OECD, 2020a; Parsons, 2020). Detailed examination of the impact of the pandemic on children living with their parents in urban areas by Yeasmin et al. (2020), showed that these children were more likely to have poorer mental health when compared to the rural area's child. Yeasmin et al. (2020) attributed this finding to the fact that children living rural areas were forced to stay indoors, whereas children in urban areas were still able to play outside and they had stronger immune systems. Therefore, being mindful of context is key to meeting the psychological needs of children during a crisis.

\section{Lockdown, Quarantine and Social Distancing}

Much of the available literature has confirmed that government-imposed lockdowns and social distance measures impacted children in different ways, often increasing the likelihood of mental health issues (Garcia de Avila et al., 2020; Urbano Agbing et al., 2020; Yeasmin et al., 2020; Yue et al., 2020). Over a sustained period, these losses are likely to negatively impact children's mental health (Courtney et al., 2020). The research is clear in this regard. Yeasmin et al. (2020) reported an increase in depression, anxiety, and sleep disturbance during lockdown amongst children living in Bangladesh. Similarly, Garcia de Avila et al. (2020) confirmed that Brazilian schoolchildren who were socially distanced from both parents, children living in households with higher number of inhabitants, children living with young parents, and parental low-education level, had lower test scores than children who were socially isolated with a person other than their parent. Together, these findings highlight the crucial human need to reconnect with others and the role parents play in children's lives, particularly in times of crises. Yue et al. (2020) reported that children confined within the home during the COVID-19 lockdown in China developed symptoms of psychological distress, including anxiety, depression and PTSD. In Parsons' (2020) study, preschool children were unable to verbally express their feelings around what was going on, mostly because they could not understand what was happening, had sleep and appetite disturbances, and also behaviour problems. In addition, Parsons (2020) reported that school children were more disorganised and exhibited more disruptive behaviour at school, and consequently their academic performance suffered. These findings suggest that while younger children tend to worry because they may find it hard to fully comprehend the situation; older children worry about their future prospects and the financial situation within the family. Nevertheless, research has confirmed that children of all ages seem to be afraid of what the coronavirus might do to them and to their loved ones (Pittinsky, 2020; Roy et al., 2020). Taken together, these studies confirmed that mental health issues remained fairly elevated among children in different countries around the world.

Even though for most children, the pandemic and school closure have led to disruptions in their routines, and social isolation may have led to increase in feelings of boredom, frustration and fear, this was not always the case. Yue et al. (2020) also reported that even though Chinese children suffered from higher levels of anxiety than their parents, they suffered significantly lower levels of PTSD than their parents during the outbreak. Such differences could be attributed the differences in cognitive abilities between children and adults, where children's cognitive abilities were still developing. However, higher levels of anxiety in children could be a result of lack of understanding of the issue and lack of coping strategies (Yue et al., 2020). Yue et al. (2020) attributed three factors to the increase in anxiety in children: first, at the time of data collection, the coronavirus outbreak was under control and this could have alleviated some of the destress caused by the pandemic; second, there has been an increases in online mental health services during quarantine which helped people cope better under these circumstances; and third, quarantine increased the time families spent together and this could have brought them closer to each other, thus improving family relationships and easing psychological distress. Similarly, in a study by Urbano Agbing et al. (2020), children in the Philippines, described the coronavirus as a deadly, dangerous, contagious, a disease which kills people worldwide; and they expressed sadness, fear, boredom, anger, disappointments about their experiences during these difficult times. Urbano Agbing et al. (2020) reported that while children expressed mixed emotions (both positive and negative) about lockdown, they also expressed hope 
and faith in God and prayers. Consequently, the children employed various coping mechanisms, such as taking up new hobbies and interests as acts of mindfulness. Urbano Agbing et al. (2020) suggested that such strategies can assist children during critical events, but the researchers also called for longitudinal research to explore the effects of pandemic, and its aftermath, on health outcomes of different children.

Despite the conflicting evidence, lockdowns and quarantine are difficult for children. In this regard, Brooks et al. (2020) suggested that whenever quarantine cannot be dismissed, it should be as brief as medically possible, and medical officials should provide clear rationale for quarantine, enough information about protocols, ensure sufficient supplies and support in order to minimise the psychological impact of quarantine and isolation. Undoubtedly, in order to reduce psychological stress for children some sort of structure or routine and social interactions are recommended (OECD, 2020a). Parents also have a pivotal role in this regard. Parents can create a learning routine at home and engage children in creative and mentally stimulating indoor activities to help them manage their anxiety and meet their emotional needs (Garcia de Avila et al., 2020; Roy et al., 2020), particularly by creating fun activities during the pandemic (Garcia de Avila et al., 2020). Engaging children with school work and chores, and allowing them enough time for daily exercise have been reported to reduce depression and anxiety (Kang et al., 2020; Yeasmin et al., 2020). Additionally, parents can find ways to help children stay in touch with friends so that they do not feel lonely and isolated.

\section{Social Media}

Measures to restrict the coronavirus transmission in the community, such as lockdowns, social distancing, and restrictions in movement, have often resulted in increased screen time, particularly for children, but not only (Roy et al., 2020). The way information about the coronavirus is presented to children can impact their ability to adjust to the situation (Fong \& Iarocci, 2020). Moreover, the younger the children are, the less likely they are able to process the information correctly and they may over- or under-estimated the threat of the virus. This means that constant misinformation on social media portals may increase the incidence of children experiencing panic and anxiety attacks (Roy et al., 2020). In fact, research confirmed that frequent social media exposure during the COVID-19 outbreak was positively associated with high prevalence of mental health issues (Gao et al., 2020; Roy et al., 2020) and put children at higher risk of experiencing anxiety and PTSD (Yue et al., 2020). Together, these findings call for a different discourse around COVID-19 and children on social media as a necessary tool in addressing fear of the coronavirus in children (Roy et al., 2020; Yue et al., 2020). More importantly, these findings highlight the need for a reduction in children's exposure to social media and negative news (Roy et al., 2020; Yue et al., 2020).

More importantly, Pittinsky (2020) cautioned that shielding children from the truth or having very high expectations of them during the pandemic could be more harmful. Parents have a role to play in this regard in that they need to monitor the children's use of social media and teach them how to rely on reliable sources of information about the pandemic. Certainly, children need to be provided with clear, accurate and meaningful explanation of the current situation. By using age-appropriate tools, vocabulary and communication strategies about the pandemic, which neither over-estimate nor minimise the dangers, parents will ensure that children do not feel guilty about the situation and so that parents do not heighten children's fears (Fong \& Iarocci, 2020; Garcia de Avila et al., 2020; OECD, 2020a; Pittinsky, 2020; Roy et al., 2020). As evidenced by an array of recent research (Garcia de Avila et al., 2020; Kang et al., 2020; Yue et al., 2020), providing children with accurate and meaningful explanations about the coronavirus may help alleviate their worries and anxiety. Nevertheless, parents need to monitor children's reactions with care and caution.

\section{Psychological Support Services}

Major events, such as the COVID-19 pandemic, influence development over the lifespan and even across generations. Therefore, the burden of the COVID-19 pandemic makes a strong case for promoting the wellbeing of children and families. Children who experience trauma during the pandemic may require a supportive structure that is closely aligned with both educational and mental health services (Firestein, 2019), a need that cannot be overlooked (Fong \& Iarocci, 2020; Sprang \& Silman, 2013). It is likely that 
interventions and strategies to address mental health issues during the pandemic (Hawke et al., 2020) and the need to strengthen families in order to help children feel safe (Garcia de Avila et al., 2020) are vital ways of supporting children's mental health and wellbeing.

In these difficult times, psychological support services are essential more than ever to detect and treat anxiety disorders and emotional distress in children (Courtney et al., 2020; Kang et al., 2020), and child maltreatment (Lawson et al., 2020; Yeasmin et al., 2020). However, psychological support services in the form of face-to-face meetings can be costly for many families who are already facing financial hardships, and availability can be problematic in face of social distancing measures (Parsons, 2020). This is especially true of low-income families who are less likely to have access psychological support services due to lack of financial resources to meet these needs. To overcome these financial constraints, governments should legislate to provide technology-based psychological interventions to children and parents, which could be a useful alternative in the current circumstances (Fong \& Iarocci, 2020). Indeed, recently developed effective approaches to providing psychological support services during the pandemic range from psychological intervention strategies (Lawson et al., 2020; Prime et al., 2020; Yeasmin et al., 2020) to telemedicine as a means of supporting the mental health needs of individuals, including children (Ashikkali et al., 2020).

Given the associated economic benefits of improved mental health issues, social and medical services, counselling and socialisation opportunities should be provided to children and their families during the pandemic (OECD, 2020a). Consequently, understanding the most effective combination of support in different population is an important next step. Next, policies need to be put in place to reduce barriers to psychological support services in order to strengthen both the children's and the family' mental health and wellbeing (Fong \& Iarocci, 2020).

\section{Limitations and Future Directions}

The limitations of this scoping review highlight the paucity in the literature related to children's mental health and wellbeing in times of global crises. Based on the evidence reviewed here, the majority of the literature reviewed targeted the COVID-19 pandemic as a medical issue rather than as an issue that could trigger mental health problems. In fact, only a limited number of studies proposed appropriate interventions to improve children's mental health and wellbeing during these difficult times.

The lack of available research suggests that there remain several aspects of the impact of the COVID19 pandemic on children's mental health and wellbeing about which relatively little is known. First, further research would benefit from intervention evaluations that provide support to children's mental health, during and after the pandemic. Second, there is a need for the exploration of new and effective strategies that help children and their families cope with the direct and indirect effects of the stress caused by the pandemic in order to ensure their wellbeing, during this and future outbreaks. Third, research to further explore the impact of the pandemic on girls' mental health and wellbeing is also required.

\section{Conclusions}

This study reviewed the results of published literature to explore the impact of the COVID-19 pandemic on children's (from birth to 17 years of age) mental health and wellbeing. Collectively, these studies indicated that globally, children have been exposed to severe and unprecedented events that have disrupted their routines. The pandemic has posed severe economic hardships on the entire planet, that have social consequences for people of all ages. Clearly, the impacts of the COVID-19 pandemic on children's holistic wellbeing are numerous and go beyond the risk of acquiring a severe acute respiratory infection. All this has repercussions for children, which if not addressed, are likely to be longstanding and could have even worse outcomes in the future. In this regard, this scoping review is an important first step towards holistically evaluating the effects of the COVID-19 pandemic on children's mental health and the resources need to help them overcome these challenges, an area in which exiting quality research is scarce (Fong \& Iarocci, 2020). This paper marks the starting point from which informed conversations around children's emotional and social wellbeing during a major crisis can evolve. 
The impact of COVID-19 on children's mental health and wellbeing...

Lastly, the findings from this scoping review have practical implications for policymakers and educators. It is recommended that governments, non-governmental organizations, educators, healthcare and psychosocial professionals adopt a collaborative approach towards preventing further mental health issues caused by the pandemic. Thus, interventions with children and families should be considered as a way of preventing the potential negative effects from the COVID-19 pandemic. To achieve this, professionals need to work with parents and children to provide them with strategies to lower the negative impact of the pandemic on their mental health. Governments, employers and policymakers clearly have a useful role to plan in financially supporting families to reduce the stress and mental health issues caused by the pandemic.

\section{Declarations}

Acknowledgements: The author would like to thank the anonymous reviewers who made comments on the paper.

Authors' contributions: This paper was written entirely by the sole author.

Competing interests: The author declared no potential conflicts of interest with respect to the research, authorship and/or publication.

Funding: The author received no financial support for the research, authorship and/or publication of this article.

\section{References}

Arksey, H., \& O'Malley, L. (2005). Scoping studies: Towards a methodological framework. International Journal of Social Research Methodology, 8(1), 19-32. https://doi.org/10.1080/1364557032000119616

Ashikkali, L., Carroll, W., \& Johnson, C. (2020). The indirect impact of COVID-19 on child health. Paediatric and Child Health, 30(12), 430-437. https://doi.org/10.1016/j.paed.2020.09.004

Bond, E.C., Dibner, K., \& Schweingruber, H. (Eds.). (2020). Reopening K-12 schools during the COVID-19 pandemic: Prioritizing health, equity, and communities. Washington, DC: The National Academies Press. https://doi.org/10.17226/25858.

Bronfenbrenner, U., \& Morris, P. A. (2006). The bioecological model of human development. In R. M. Lerner, \& W. Damon (Eds.), Handbook of child psychology. Theoretical models of human development (6 ${ }^{\text {th }}$ ed., pp. 793-828). Hobanken, New Jersey: John Wiley \& Sons, Inc.

Brooks, S.K., Webster, R.K., Smith, L.E., Woodland, L., Wessely, S., Greenberg, N., ... (2020). The psychological impact of quarantine and how to reduce it: rapid review of the evidence. Lancet. 395(10227), 912-920. https://doi.org/10.1016/S0140-6736(20)30460$\underline{8}$

Castagnoli, R., Votto, M., Licari, A., Brambilla, I., Bruno, R., Perlini, S., ... Marseglia, G.L. (2020). Severe acute respiratory syndrome coronavirus 2 (SARS-CoV-2) infection in children and adolescents: A systematic review. JAMA Pediatr. 174(9), 882-889. https://doi.org/10.1001/jamapediatrics.2020.1467

Courtney, D., Watson, P., Battaglia, M., Mulsant, B.H., \& Szatmar, P. (2020). COVID-19 impacts on child and youth anxiety and depression: Challenges and opportunities. The Canadian Jounal of Psychiatry, 65(1), 688-691. https://doi.org/10.1177/0706743720935646

Firestein, C. (2019, June). Advising students who struggle due to traumatic events. Academic Advising Today, 42(2). Retrieved from https://nacada.ksu.edu/Resources/Academic-Advising-Today/View-Articles/Advising-Students-Who-Struggle-Due-ToTraumatic-Events.aspx

Fong, V.C., \& Iarocci, G. (2020). Child and family outcomes following pandemics: A systematic review and recommendations on COVID-19 policies. Journal of Pediatric Psychology, 45(10), 1124-1143. https://doi.org/10.1093/jpepsy/jsaa092

Gao, J., Zheng, P., Jia, Y., Chen, H., Mao, Y., Wang, Y.,... Dai, J. (2020). Mental health problems and social media exposure during COVID-19 outbreak. PLoS ONE, 15(4), e0231924. https://doi.org/10.1371/journal.pone.0231924

Garcia de Avila, M.A., Hamamoto Filho, P.T., da Silva Jacob, F.L., Souza Alcantara, L.R., Berghammer, M., Nolbris, M.J., ... Nilsson, S. (2020). Children's anxiety and factors related to the COVID-19 pandemic: An exploratory study using the children's anxiety questionnaire and the numerical rating scale. International Journal of Environmental Research and Public Health, 17(16), 5757. https://doi.org/10.3390/ijerph17165757

Grant, M. J., \& Booth, A. (2009). A typology of reviews: An analysis of 14 review types and associated methodologies. Health Information $\mathcal{E}$ Libraries Journal, 26(2), 91-108. https://doi.org/10. 1111/j.1471-1842.2009.00848.x 


\section{Jane SPITERI}

Gromada, A., Rees, G., \& Chzhen, Y. (2020). Worlds of Influence: Understanding What Shapes Child Well-being in Rich Countries, Innocenti Report Card no. 16. Florence: UNICEF Office of Research - Innocenti. Retrieved from https://www.unicefirc.org/publications/1140-worlds-of-influence-understanding-what-shapes-child-well-being-in-rich-countries.html

Hawke, L.D., Barbic, S.P., Voineskos, A., Szatmari, P., Cleverley, K., Hayes, E.,... Henderson, J.L. (2020). Impacts of COVID-19 on youth mental health, substance use, and well-being: A rapid survey of clinical and community samples. The Canadian Journal of Psychiatry, 65(10), 701-709. https://doi.org/10.1177/0706743720940562

Infurna, F. J., \& Luthar, S. S. (2016). Resilience to major life stressors is not as common as thought. Perspectives on Psychological Science, 11(2), 175-194. https://doi.org/10.1177/1745691615621271

Kang, Y. Q., Lim, T. S. H., Ragen, E.S., Tan, M. Y., \& Aishworiya, R. (2020). Managing children's anxiety during COVID-19 pandemic: Strategies for providers and caregivers. Frontiers in Psychiatry, 11, 552823. https://doi.org/10.3389/fpsyt.2020.552823

Keshri, A.K. (2020). COVID-19 reminds us why we must invest more on family-based alternatives for children. Institutionalised Children Explorations and Beyond, 1(8), 133-140. https://doi.org/10.1177/2349300320970762

Lawson, M., Piel, M.H., \& Simon, M. (2020). Child Maltreatment during the COVID-19 pandemic: Consequences of parental job loss on psychological and physical abuse towards children. Child Abuse $\mathcal{E}$ Neglect, 110, 104709. https://doi.org/10.1016/j.chiabu.2020.104709

Levac, D., Colquhoun, H., \& O’Brien, K.K. (2010). Scoping studies: Advancing the methodology. Implementation Science, 5(1), 1-9. https://doi.org/10.1186/1748-5908-5-69

Ludvigsson, J.F. (2020). Systematic review of COVID-19 in children shows milder cases and a better prognosis than adults. Acta Paediatr, 109(6), 1088-1095. https://doi.org/10.1111/apa.15270

Martin, J., McBride, T., Masterman, T., Pote, I., Mokhtar, N., Oprea, E., \& Sorgenfrei, M. (2020). Covid-19 and early intervention: Evidence, challenges and risks relating to virtual and digital delivery. London: Early Intervention Foundation.

Organisation for Economic Co-operation and Development [OECD]. (2020a). The impact of COVID-19 on student equity and inclusion: Supporting vulnerable students during school closures and school re-openings. Paris: OECD Publishing. Retrieved from http://www.oecd.org/coronavirus/policy-responses/the-impact-of-covid-19-on-student-equity-and-inclusion-supportingvulnerable-students-during-school-closures-and-school-re-openings-d593b5c8/

Organisation for Economic Co-operation and Development [OECD]. (2020b). Teaching in focus \#32. How prepared are teachers and schools to face the challenges to learning caused by the coronavirus pandemic?. Paris: OECD Publishing. Retrieved from https://www.oecdilibrary.org/docserver/2fe27ad7en.pdf?expires=1591790477\&id=id\&accname=guest\&checksum=24B9F5B223ABFD0884EBA56EAFC2732C

Papetti, L., Di Loro, P.A., Tarantino, S., Grazzi, L., Guidetti, V., Parisi, P., ... Valeriani1, M.; on behalf of the Italian Headache Society (SISC) specific interest group on pediatric headaches. (2020). I stay at home with headache. A survey to investigate how the lockdown for COVID-19 impacted on headache in Italian children. Cephalagia, 40(13), 1459-1473. https://doi.org/10.1177/0333102420965139

Parsons, J. (2020). COVID-19, children and anxiety in 2020. The Royal Australian College of General Practitioners, 49 Suppl 27. https://doi.org/10.31128/AJGP-COVID-27

Pittinsky, R. (2020). Managing COVID-19 anxiety. Phi Delta Kappan, 102(3), 68-68. https://doi.org/10.1177/0031721720970709

Prime, H., Wade, M., \& Browne, D.T. (2020). Risk and resilience in family well-being during the COVID-19 pandemic. American Psychologist, 75(5), 631-643. https://doi.org/10.1037/amp0000660

Roy, A., Singh, A.K., Mishra, S., Chinnadurai, A., Mitra, A., \& Bakshi, O. (2020). Mental health implication of COVID-19 pandemic and its response in India. International Journal of Social Psychiatry, 1-14. https://doi.org/10.1177/0020764020950769

Spaull, N. (2020). COVID-19 and schooling in South Africa: Who should go back to school first? Prospects. https://doi.org/10.1007/s11125-020-09470-5

Sprang, G., \& Silman, M. (2013). Posttraumatic stress disorder in parents and youth after health-related disasters. Disaster Medicine and Public Health Preparedness, 7(1), 105-110. https://doi.org/10.1017/dmp.2013.22

United Nations Educational, Scientific and Cultural Organization [UNESCO]. (2020a). Three ways to plan for equity during the school closures. Geneva: United Nations. Retrieved from https://gemreportunesco.wordpress.com/2020/03/25/three-ways-to-planfor-equity-during-the-coronavirus-school-closures/

United Nations Educational, Scientific and Cultural Organization [UNESCO]. (2020c). COVID-19 Educational disruption and response. Retrieved from https://en.unesco.org/covid19/educationresponse.

United Nations Educational, Scientific and Cultural Organization [UNESCO]. (2020d). What price will education pay for COVID-19? Paris: UNESCO. Retrieved from http://www.iiep.unesco.org/en/what-price-will-education-pay-covid-19-13366 
The impact of COVID-19 on children's mental health and wellbeing...

United Nations Educational, Scientific and Cultural Organization[ UNESCO]. (2020b). 10 recommendations to ensure that learning remains uninterrupted. Retrieved from https://en.unesco.org/news/covid-19-10-recommendations-plan-distancelearningsolutions.

United Nations. (2020). Policy brief: The impact of COVID-19 on children. Geneva: United Nations. Retrieved from https://www.un.org/sites/un2.un.org/files/policy brief on covid impact on children 16 april 2020.pdf

Urbano Agbing, L., Agapito, J.D., Albano Baradi, A.M. Guzman, M.B.C., Mariano Ligon, C., \& Tuazon Lozano, A. (2020). COVID-19: In the eyes of a Filipino child. Illness, Crisis $\mathcal{E}$ Loss, 1-20. https://doi.org/10.1177/1054137320966846

World Health Organization. (2017). Depression and other common mental disorders: Global health estimates. Geneva, Switzerland: WHO Document Production Services. Geneva: World Health Organization. Retrieved from https://apps.who.int/iris/bitstream/handle/10665/254610/WHO-MSD-MER-2017.2-eng.pdf

World Health Organization. (2020). Addressing violence against children, women and older people during the COVID-19 Pandemic: Key actions. World Health Organization. Geneva: World Health Organization. Retrieved from https://www.who.int/publications/i/item/WHO-2019-nCoV-Violence actions-2020.1

Xie, X., Xue, Q., Zhou, Y., Zhu, K., Liu, Q., Zhang, J., \& Song, R. (2020). Mental health status among children in home confinement during the coronavirus disease 2019 outbreak in Hubei Province, China. JAMA Pediatrics, 174(9),898-900. https://doi.org/10.1001/jamapediatrics.2020.1619

Yeasmin, S., Banik, R., Hossain, S.I., Hossain, N., Mahumud, R., Salma, N., \& Hossain, M. (2020). Impact of COVID-19 pandemic on the mental health of children in Bangladesh: A cross-sectional study. Children and Youth Services Review, 117, 105277. https://doi.org/10.1016/j.childyouth.2020.105277

Yue, J., Zang, X., Le, Y., \& An, Y. (2020). Anxiety, depression and PTSD among children and their parent during 2019 novel coronavirus disease (COVID-19) outbreak in China. Current Psychology. https://doi.org/10.1007/s12144-020-01191-4 